\title{
Modulation of fat metabolism and gut microbiota by resveratrol on high-fat diet-induced obese
} mice

\author{
C Linda Campbell ${ }^{1,2, *}$ \\ Renqiang $\mathrm{Yu}^{3,4}$ \\ Fengzhi Li' \\ Qin Zhou ${ }^{3}$ \\ Daozhen Chen ${ }^{4}$ \\ Ce Qi ${ }^{1,2}$ \\ Yongxiang Yin $^{5}$ \\ Jin $\operatorname{Sun}^{1,2, *}$
}

'State Key Laboratory of Food Science and Technology, Jiangnan University, Wuxi 214122, China;

${ }^{2}$ School of Food Science and

Technology, Jiangnan University,

Wuxi $2|4| 22$, China; ${ }^{3}$ Department

of Neonatology, The Affiliated

Wuxi Maternity and Child Health

Care Hospital of Nanjing Medical

University, Wuxi 214002, China;

${ }^{4}$ Department of Central Lab, The

Affiliated Wuxi Maternity and Child

Health Care Hospital of Nanjing

Medical University, Wuxi 214002,

China; ${ }^{5}$ Department of Pathology,

The Affiliated Wuxi Maternity and

Child Health Care Hospital of

Nanjing Medical University, Wuxi

2/4002, China

*These authors contributed equally to this work

Correspondence: Renqiang Yu;

Daozhen Chen

The Affiliated Wuxi Maternity and Child Health Care Hospital of Nanjing Medical University, 48 Huaishu Lane, Wuxi

2I 4002, PR China

$\mathrm{Tel}+8651082725161$

Fax +8651082725094

Email yurenqiang@njmu.edu.cn;

chendaozhen@।63.com
This article was published in the following Dove Medical Press journal:

Diabetes, Metabolic Syndrome and Obesity:Targets and Therapy

Purpose: The antioxidant resveratrol (RSV) has low bioavailability and can reach the colon to access the gut microbial ecosystem. RSV administration together with high-fat diet prevented abnormal changes of intestinal microbiota. However, whether or not RSV can reshape the intestinal microbiota of obese mice and alleviate obesity-related diseases remains to be studied. This study aimed to explore the role of RSV in alleviating high-fat-induced obesity and its relationship with oxidative stress and gut microbiota.

Methods: Male C57BL/6 mice were divided into five groups and administered for 16 weeks with: standard diet (CON), high-fat diet (60\% energy for lard, HFD), and HFD with low, medium, and high dose of RSV, 50, 75, and $100 \mathrm{mg} / \mathrm{kg}$ body weight administered daily via drinking water, respectively.

Results: Medium and high RSV treatment significantly prevented body weight gain, decreased relative weight of liver and adipose tissue compared with HFD $(P<0.05)$. All doses significantly prevented HFD-induced increase of serum triglyceride, low density lipoprotein cholesterol, glucose, and endotoxemia $(P<0.05)$. Medium and high dose also prevented chronic inflammation by decreasing serum interleukin- 1 and tumor necrosis factor-alpha $(P<0.05)$, and oxidative stress in liver and brain indicated by increase in superoxide dismutase, catalase, glutathione peroxidase activity $(P<0.05)$. Formation of malondialdehyde was prevented by all doses compared with HFD $(P<0.05)$. Both medium and high doses of RES increased alpha diversity of gut microbiota according to the Chaol and Shannon indices $(P<0.05)$. Medium dose induced obvious shift in gut microbiota composition according to principal component analysis. High dose of RSV effectively prevented HFD-induced increase of Coriobacteriaceae and Desulfovibrionaceae $(P<0.05)$, which show a significant correlation with body weight $(r>0.8 \quad P<0.00)$.

Conclusion: RSV prevented HFD-induced endotoxemia, oxidative stress, and gut microbiota change.

Keywords: resveratrol, high fat diet, obesity, oxidative stress, gut microbiota

\section{Introduction}

Obesity has been a growing health concern over the past few decades in industrialized and developing countries alike. ${ }^{1,2}$ Studies have shown that obesity contributes significantly to major health problems worldwide ranging from atherosclerosis, hypertension, metabolic syndrome, stroke, heart diseases, and even specific forms of cancer. ${ }^{3}$ Abdominal obesity is associated with dyslipidemia characterized by increasing blood triglyceride (TG) and decreasing high-density lipoprotein cholesterol (HDL-C). It is known that long-term feeding on a high-fat diet (HFD) can induce obesity with 
hyperphagia, hyperglycemia, hyperlipidemia, and insulin resistance in mice. ${ }^{4}$ It has been reported that HFD-induced disequilibrium in the gut microbiota leads to increased oxidative stress via stimulation of endotoxin secretion and weakening of the gut-barrier protection. ${ }^{5}$ HFD increases ROS in liver and adipose tissues. The latter leads to the reduction of mucosa protection and increases exposure to oxidative stress. ${ }^{6}$ Concomitant to the aforementioned phenomenon, the percentage of Bifidobacteria and Lactobacilli in the gut microbiota is reduced while harmful bacteria adhere to the intestinal epithelium leading to loss of gut function., ${ }^{7,8}$

Resveratrol (RSV) (3, 5, 4'-trihydroxystilbene) belongs to a large group of polyphenols found in several groups of plants. The richest natural source of RSV is the Polygonum cuspidatum plant, although significant amounts have been found in peanuts, grapes, and red wine. ${ }^{9,10}$ Besides its natural form, this compound is available in tablets and it is recommended as a dietary supplement., ${ }^{9,11}$ RSV has substantially increased in recent years, because of its broad biological activity shown in the following: Anti-obesity, anti-diabetes, improving gut microbes, antioxidant leading to the decrease of free radicals. ${ }^{12,13}$

RSV has been shown to exert anti-obesity-related disease, including diabetic and dyslipidemia via multiple mechanisms, including anti-inflammation and antioxidant effects. ${ }^{14-16}$ RSV showed poor water solubility, which is likely responsible for the low oral bioavailability in vivo. Its metabolites in the circulation are excreted back into the intestines presumably via biliary secretion. ${ }^{17}$ It was reported that oral administration of RSV at daily doses of 0.5 and $1.0 \mathrm{~g}$ in human can achieve sufficient levels in the gastrointestinal tract. Orally administered RSV can reach the colon due low bioavailability, increasing its potential to interact with the gut microbiota. It has been reported that trans-RSV supplementation alone or in combination with quercetin scarcely counteracts gut microbiota dysbiosis produced by high-fat sucrose diet in mouse. ${ }^{18}$ It is reported recently that RSV inhibited gut production of trimethylamine, a precursor of trimethylamine-N-oxide (TMAO) which could enhance atherosclerosis, via gut microbiota remodeling in mice. It also increased gut Lactobacillus and Bifidobacterium with bile salt hydrolase activity, thereby enhanced bile acid de-conjugation and fecal excretion. ${ }^{19}$ Another recent study indicated that RSV administration to obese mice could reduce the relative abundances of Turicibacteraceae, Moryella, Lachnospiraceae, and Akkermansia, and increase those of Bacteroides and Parabacteroides. The study also found improved glucose homeostasis in mice after fecal transferring from healthy RSV-administrated donor mice. ${ }^{20,21}$

In human study, it was also confirmed that combined epigallocatechin-3-gallate and RSV supplementation for 12 weeks could significantly reduce the abundance of Bacteroidetes and tended to decrease the abundance of Faecalibacterium prausnitzii in overweight men. ${ }^{22}$ We previously confirmed that RSV administration together with HFD leads to marked changes in the composition of the gut microbiota in obese mice. ${ }^{23}$ However, whether or not RSV can reshape the intestinal microbiota of obese mice and alleviate obesityrelated diseases remains to be studied.

In this study, we evaluated the effect of RSV on the gut microbiota of HFD-induced obese mice, by introducing RSV through their drinking water. We postulate that the beneficial effect of RSV may be imparted by its direct effect on the gut barrier system.

\section{Materials and methods Diets and animals}

Sixty male C57BL/6J mice (4-week old) were obtained from Suzhou Grareway Biotechnology Limited (Suzhou, China), and kept in an environmentally controlled breeding room (temperature: $23^{\circ} \mathrm{C} \pm 2^{\circ} \mathrm{C}$, humidity: $60 \% \pm 5 \%$, a regular 12 -hour light-dark cycle).

After 1-week acclimation on a chow diet, the animals were randomly assigned into five groups: 1) Normal chow diet (NC); 2) High fat-diet (HFD, Research Diets D12492, Table 1); 3) HFD with low (HFD-L, $50 \mathrm{mg} / \mathrm{kg}$ body weight daily), medium (HFD-M, $75 \mathrm{mg} / \mathrm{kg}$ body weight daily), and high (HFD-H, $100 \mathrm{mg} / \mathrm{kg}$ body weight daily) RSV (Solarbio, Beijing, China) treatment. RSV were dissolved in $0.4 \mathrm{~mL}$ of absolute ethanol and added to $100 \mathrm{~mL}$ of drinking water daily for a period of 3 months whereas, NC and HFD received $0.4 \%$ of absolute ethanol added to $100 \mathrm{~mL}$ of drinking water for the same period of time. All mice had ad libitum access to water and food throughout the study. Feces were collected weekly and stored at $-80^{\circ} \mathrm{C}$ for analysis. The following were also collected and stored at $-80^{\circ} \mathrm{C}$ : ileum, liver, epididymal, mesenteric, perinephric fat, blood, and colon for further analysis. Mice were sacrificed by cervical dislocation after 8 hours of fasting period.

\section{Biochemical analysis}

Blood samples were collected, quarantined, and centrifuged after a 6-hour fasting period at $1,500 \times g$ and at $4^{\circ} \mathrm{C}$ for 10 
Table I Ingredient composition of the diets fed to mice

\begin{tabular}{|l|l|l|}
\hline Ingredients & $\begin{array}{l}\text { High fat diet } \\
\text { (D I 2492 g \%) }\end{array}$ & $\begin{array}{l}\text { Normal diet } \\
\text { (D I 2450) g \%) }\end{array}$ \\
\hline Protein & 26 & 19.2 \\
\hline Carbohydrate & 26 & 67.3 \\
\hline Fat & 35 & 4.3 \\
\hline Casein & 200 & 200 \\
\hline L-cystine & 3 & 3 \\
\hline Corn starch & 0 & 506.2 \\
\hline Maltodextrin & 125 & 125 \\
\hline Sucrose & 68.8 & 68.8 \\
\hline Cellulose & 50 & 50 \\
\hline Soybean oil & 25 & 25 \\
\hline Lard & 245 & 20 \\
\hline Mineral mix SI0026 & 10 & 10 \\
\hline Dicalcium phosphate & 13 & 13 \\
\hline Calcium carbonate & 5.5 & 5.5 \\
\hline Potassium citrate, I $\mathrm{H}_{2} \mathrm{O}$ & 16.5 & 16.5 \\
\hline Vitamin mix VI000 I & 10 & 10 \\
\hline Choline bitartrate & 2 & 2 \\
\hline
\end{tabular}

minutes. Plasma total cholesterol (TC), TG, low-density lipoprotein cholesterol (LDL-C), and HDL-C content were examined using the corresponding enzymatic colorimetric assay kits (Nanjing Jiancheng Bioengineering Institute, Nanjing, China) following manufacturer's instructions. Blood glucose was measured using a glucometer (Accu-Check; Roche Diagnostics, Madrid, Spain).

\section{Assessment of antioxidant status in brain and liver}

Malondialdehyde (MDA), catalase (CAT) activity, total antioxidant capacity (TAOC), glutathione peroxidase (GSH-Px) activity, and superoxide dismutase (SOD) activity were measured. They were measured using diagnostic kit from Nanjing Jiancheng Bioengineering Institute according to the instructions.

\section{ELISA}

Interleukin (IL)-1, IL-10, Lipopolysaccharide (LPS), LPSbinding protein (LBP), and tumor necrosis factor (TNF)- $\alpha$ levels in plasma were examined by ELISA kits, and measured at $450 \mathrm{~nm}$ on Biocell HT1 ELISA microplate reader, following the manufacturer's protocol (Jiancheng Bioengineering Institute, Nanjing, China). The least noticeable concentrations were $25 \mathrm{pg} / \mathrm{mL}$ for TNF- $\alpha, 3 \mathrm{pg} / \mathrm{mL}$ for LBP and LPS, $3 \mathrm{pg} /$ $\mathrm{mL}$ for IL-10, and $3.5 \mathrm{pg} / \mathrm{mL}$ for IL-1. The coefficients of variation for all ELISA were $<5 \%$. All samples were measured in duplicate.

\section{Histological analysis (H\&E)}

The liver and epididymal fat were washed with PBS, fixed in $10 \%$ formalin solution for 15 minutes at $4{ }^{\circ} \mathrm{C}$ and stained with H\&E to observe the effect of RSV on lipid accumulation. The tissue samples were photographed $(\times 400$ original magnifications) under a microscope (DP73, OLYMPUS Co., Ltd., Tokyo, Japan).

\section{Analysis of community composition by I6S rRNA gene amplicon sequencing}

Total genomic DNA was extracted from cecal content samples using a soil DNA extraction kit (Mo Bio Laboratories, Carlsbad, CA, USA) following the manufacturer's protocol. PCR amplicon libraries were constructed for Illumina MiSeq sequencing using bacterial primers 515F (5'-GTGCCA GCMGCCGCGG-3) and 806R (5'-GGACTACHVGGG TWTCTAAT-3') targeting V4 hypervariable regions of the $16 \mathrm{~S}$ rRNA genes..$^{24}$ Each $20 \mu \mathrm{L}$ reaction mixture included $5 \times$ FastPfu Buffer, $2.5 \mathrm{mM}$ dNTPs, FastPfu Polymerase, 5 $\mu \mathrm{M}$ of each primer, and $10 \mathrm{ng}$ of template DNA. The PCR profile was set as follows: $95^{\circ} \mathrm{C}$ for 5 minutes and 27 cycles at $95^{\circ} \mathrm{C}$ for 30 seconds, $55^{\circ} \mathrm{C}$ for 30 seconds, and $72^{\circ} \mathrm{C}$ for 45 seconds, with a final extension at $72^{\circ} \mathrm{C}$ for 10 minutes. Reads from the original DNA fragments were merged using FLASH (http://ccb. jhu.edu/software/FLASH/), ${ }^{25}$ and quality filtering was performed based on previously published data. ${ }^{26}$ Sequencing data were processed using the Quantitative Insights into Microbial Ecology (QIIME) pipeline. Operational Taxonomic Units (OTUs) were picked using de novo OTU picking protocol with a $97 \%$ similarity threshold. RDP classifier was used to taxonomically annotate these sequences. ${ }^{27}$ The microbial diversity was analyzed using the QIIME software with Python scripts. Alpha diversity analysis included Shannon index, Chao1, and observed species. Jackknifed beta diversity included both unweighted and weighted Unifrac distances calculated by subsampling 10 times, and the distances were visualized by Principal coordinate analysis (PCoA). Taxonomy assignment of OTUs was performed by comparing sequences to the Greengenes database (gg_13_5_otus).

\section{Statistical analyses}

All results are expressed as means $\pm \mathrm{SD}$; groups were performed with SPSS 22 (SPSS, Inc, Chicago, IL, USA). Differences between two groups were analyzed by one-way ANOVA test followed by the Tukey's test. When variances were not homogeneous, data were analyzed by the non-parametric 
Kruskal-Wallis test. Confidence levels for statistical significance were set at $P<0.05$.

\section{Ethics statement}

Animal experiments were carried out based on the recommendations of the Guide for the Care and Use of Laboratory Animals of the Institutional Animal Care and Use Committee of Jiangnan University and approved by the Institutional Animal Care and Use Committee of Jiangnan University.

\section{Results}

\section{RSV treatment and food intake and body}

\section{weight in mice}

We observed significant increase in body weight after 8 weeks of HFD diet $(P<0.05)$. Figure 1A shows the increase in body weight; however, medium- and high-dose treatment significantly decreased the final weight and growth rate (Figure 1B, C). The RSV-administered mice showed no difference in HFD intake (Figure 1D).
RSV treatment and histological analysis of lipids

HFD-fed mice showed significantly greater accumulation of white adipose tissue, which was prevented by medium $(P<0.05)$ and high dose $(P<0.01)$ of RSV treatment for epididymal, perinephric, mesenteric, and liver tissues (Figure 2A-D).

Histological analysis revealed that RSV prevented the deposit formation of lipid droplets in all the spaces in HFD-H mice. Mice in HFD group had larger adipocytes than those fed with the NC diet. Furthermore, RSV treatment showed that mice fed with HFD had decreased number of adipocytes, as confirmed by $\mathrm{H} \& \mathrm{E}$ stain $(P<0.05$, Figure $2 \mathrm{E})$. This clearly shows the anti-obesity activity of RSV $(P<0.05$, Figure $2 \mathrm{E})$.

\section{RSV treatment and plasma lipid}

The serum LDL-C, TC, and glucose were significantly increased in HFD-fed compared with NC group $(P<0.05)$. They were significantly decreased by RVS treatment in a dose-dependent manner $(P<0.05$ Figure $3 \mathrm{~A}, \mathrm{C}, \mathrm{D})$. Additionally, HDL-C expectedly decreased in HFD mice while
A

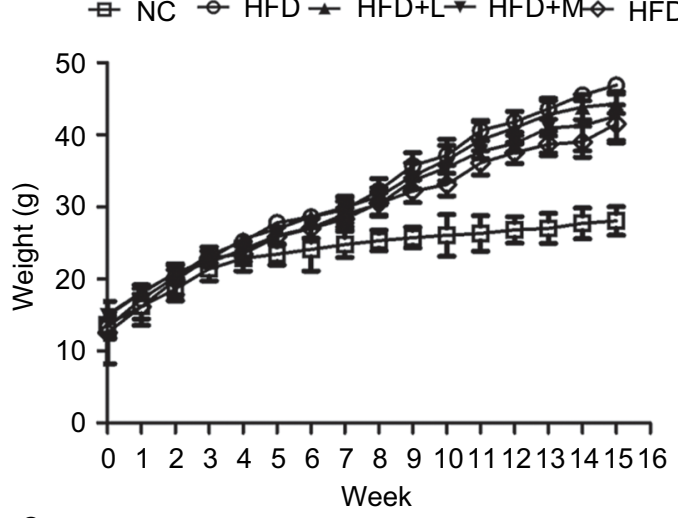

C

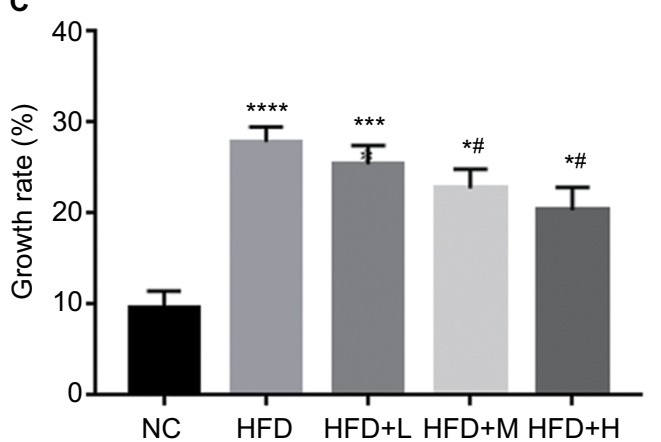

B

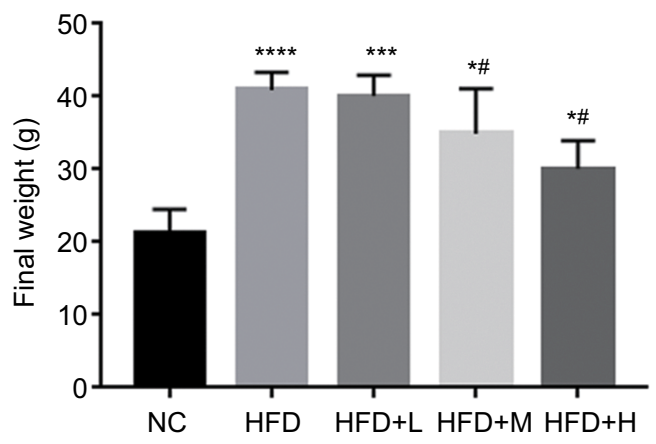

D

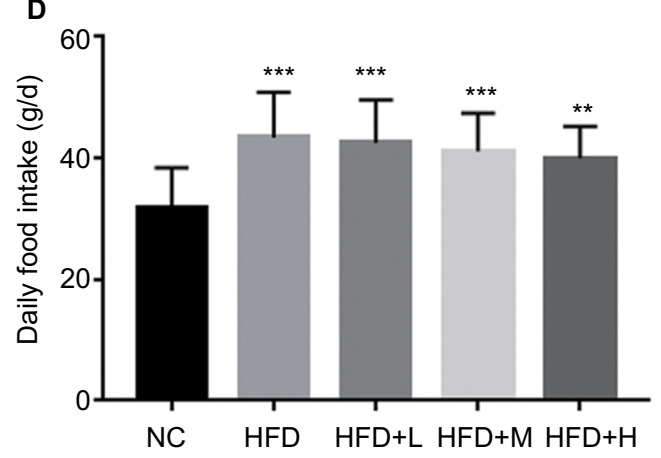

Figure I Phenotypes of mouse groups fed on different diets.

Notes: $(\mathbf{A})$ Weight $(\mathbf{g}),(\mathbf{B})$ body weight $(\mathbf{g}),(\mathbf{C})$ growth rate $(\%)$, and (D) daily food intake $(\mathrm{g} / \mathrm{d})$. Data are presented as mean \pm standard error $(\mathrm{n}=12)$. Mean values were significantly different compared with those of the NC group: ${ }^{*} P<0.05$. $* * * P<0.00 \mathrm{I}$, $* * * * P<0.000 \mathrm{I}$. Mean values were significantly different compared with those of the $\mathrm{HF}$ group: $\# P<0.05$. There were no significant differences in daily food intake among HFD-fed mice but changes were seen in NC and HFD-fed mice.

Abbreviations: HFD, high-fat diet; HFD+H, mice fed with HFD supplemented with high RSV; HFD+L, mice fed with HFD supplemented with low RSV; HFD+M, mice fed with HFD supplemented with medium RSV; NC, normal chow diet-fed mice; RSV, resveratrol. 

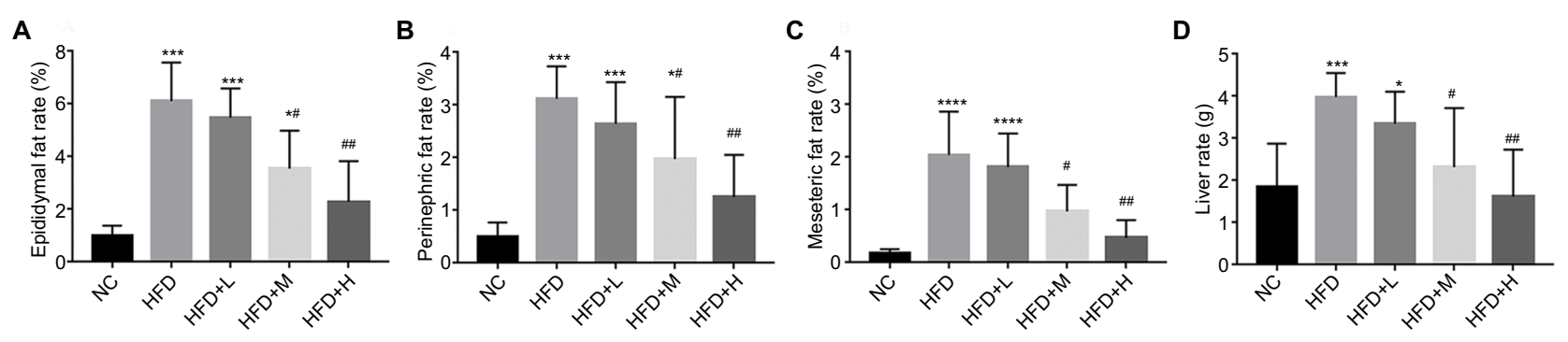

E
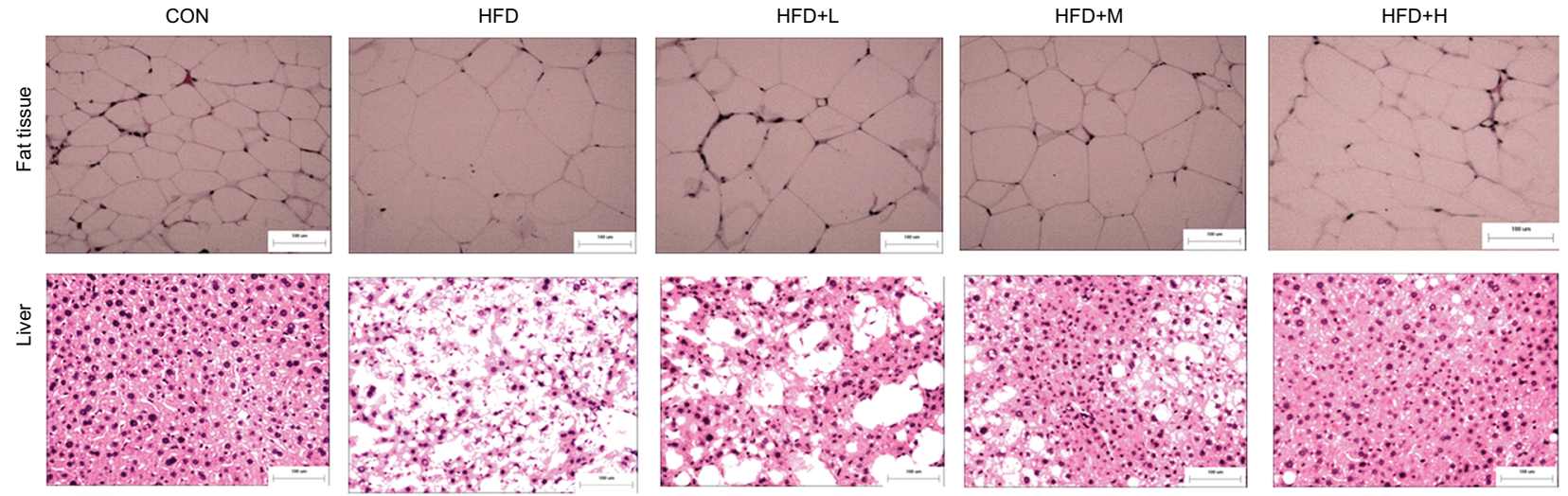

Figure 2 RSV reduced fat tissue in C57BL/6] HFD-fed mice.

Notes: (A) Epididymal fat (\%), (B) perinephric fat (\%), (C) mesenteric fat (\%), (D) liver rate, and (E) fat and liver tissue ( $\mathrm{n}=12$ per group). $* P<0.05$ and $* * P<0.0 \mathrm{I}$ compared with the NC group; ${ }^{\# P}<0.05$ and ${ }^{\# P}<0.01$ compared with the HFD control group. $* * * P<0.00$ I, $* * * * P<0.000$ I.

Abbreviations: HFD, high-fat diet; HFD+H, mice fed with HFD supplemented with high RSV; HFD+L, mice fed with HFD supplemented with low RSV; HFD+M, mice fed with HFD supplemented with medium RSV; RSV, resveratrol; NC, normal chow diet-fed mice.
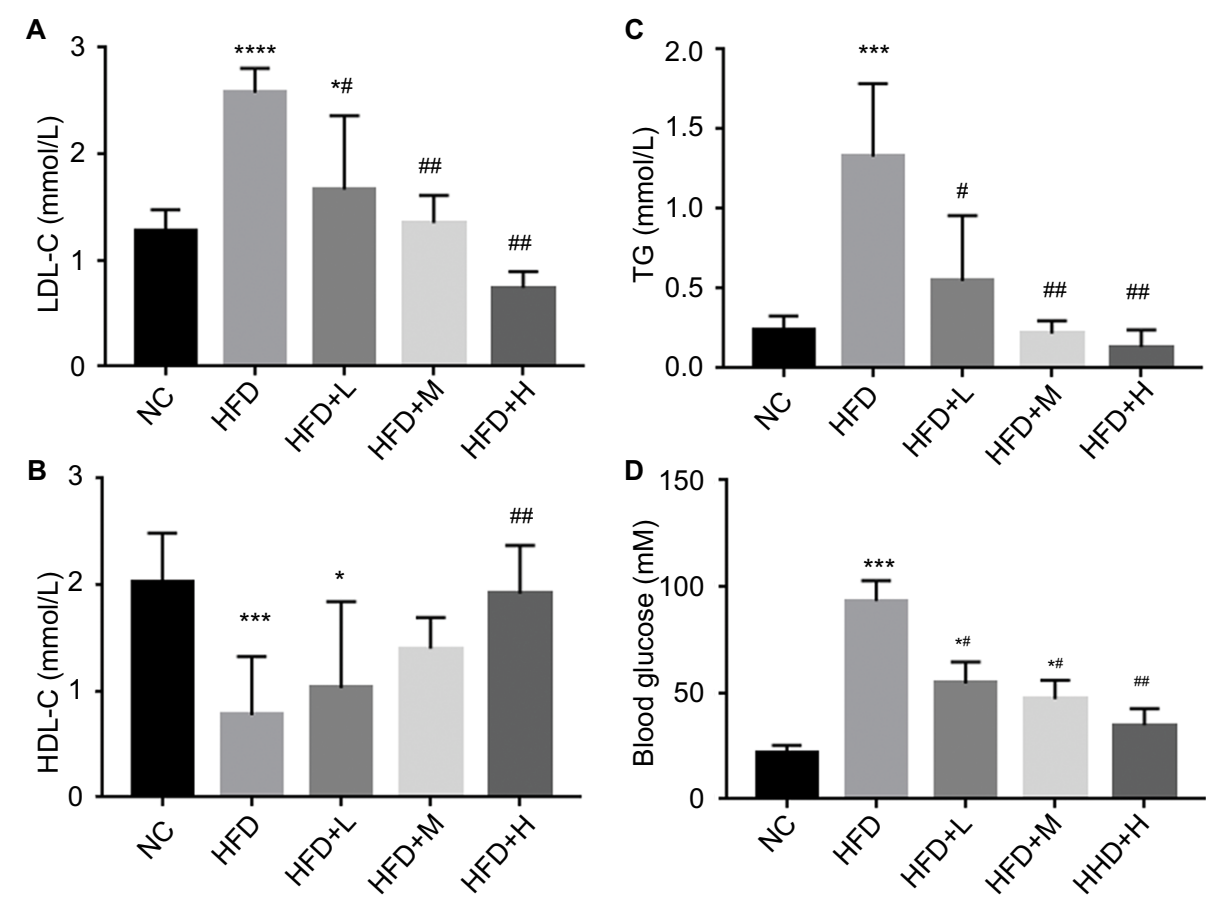

Figure 3 RSV improved serum parameters in HFD-fed mice.

Notes: (A) LDL-C (mmol/L), (B) HDL-C (mmol/L), (C) TG (mmol/L), and (D) blood glucose $(\mathrm{Mm}), \mathrm{n}=12$ per group. $* P<0.05$ and $* * P<0.01$ compared with the NC control group; ${ }^{\# P}<0.05$ and ${ }^{\# P} P<0.01$ compared with the HFD control group. $* * * P<0.001$, $* * * * P<0.0001$.

Abbreviations: HDL-C, high-density lipoprotein cholesterol; HFD, high-fat diet; HFD+H, mice fed with HFD supplemented with high RSV; HFD+L, mice fed with HFD supplemented with low RSV; HFD+M, mice fed with HFD supplemented with medium RSV; LDL-C, low-density lipoprotein cholesterol; NC, normal chow diet-fed mice; RSV, resveratrol; TG, triglyceride. 
high-dose RSV supplementation resulted in significant increase $(P<0.05$ Figure 3B).

\section{Effect of RSV treatment on HFD-induced endotoxemia and chronic inflammation}

We also evaluated anti-inflammatory ability of RSV in obese mice. We observed a significant increase in LPS, LBP, IL-1, and IL-10 levels in HFD compared with the NC group, implying the occurrence of endotoxemia and systemic chronic inflammation $(P<0.05)$. Those in the RSV group showed significantly lower levels compared with the HFC group, but were not restored to the levels observed in the NC group $(P<0.05$, Figure $4 \mathrm{~A}-\mathrm{D})$.

Mouse serum was evaluated for the levels of TNF- $\alpha$ after oral treatment of RSV. We observed a significant increase in TNF- $\alpha$ in the HFD group compared with the NC mice that was alleviated in the RSV-treated mice serum $(P<0.05$, Figure 4E).

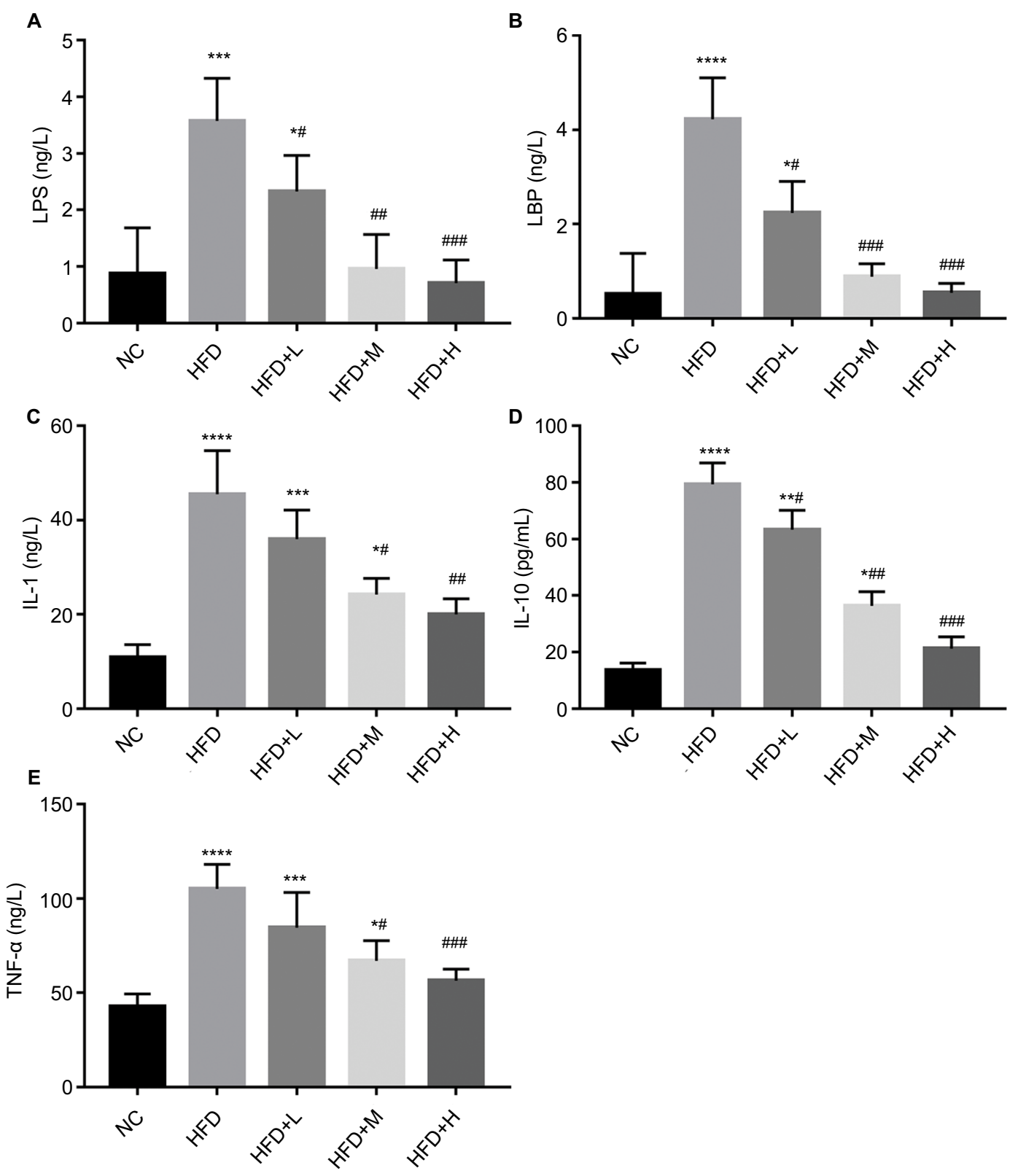

Figure 4 RSV supplements improved tissue damage, reduced endotoxemia, and inhibited the proliferation of inflammatory cytokines in the mucosa colon of HFD-fed mice. Notes: (A) LPS (ng/L), (B) LBP (ng/L), (C) IL-I (ng/L), (D) IL-I0 (pg/ml), and (E) TNF- $\alpha(\mathrm{ng} / \mathrm{L})$. Data are mean \pm SD ( $\mathrm{n}=12)$. Significant differences are indicated: $* P<0.05$; $* * P<0.01$. **** $P<0.001 ; * * * * P<0.0001$; \#P<0.05; \#\#P<0.01; \#\#P<0.00I compared with the HFD control group.

Abbreviations: HFD, high-fat diet; HFD+H, mice fed with HFD supplemented with high RSV; HFD+L, mice fed with HFD supplemented with low RSV; HFD+M, mice fed with HFD supplemented with medium RSV; IL, interleukin; LBP; LPS-binding protein; LPS, lipopolysaccharide; NC, normal chow diet-fed mice; RSV, resveratrol; TNF- $\alpha$, tumor necrosis factor alpha. 


\section{RSV treatment and oxidation in mice}

We also investigated the anti-oxidative effect of RSV in obese mice, using oxidative markers GSH-Px, CAT, and SOD, in three different categories, namely: (A) brain, (B) liver, and (C) serum. Treatment with HFD for 16 weeks weakened the antioxidant defenses of mice, with a maximum effect in the HFD group compared with NC. HFD mice showed a decreased level of oxidation in brain but increase in MDA level compared with the NC mice $(P<0.05)$. We observed significant increases in GSH-Px, CAT, and SOD with significantly reduced MDA production with multiple doses of RSV (Figure 5A). Our results also displayed a similar trend in liver and serum analysis that showed the anti-oxidative effect of RSV in HFD-fed mice (Figure 5B, C).

\section{Gut microbiota composition}

The microbiota composition was studied for cecal content by Miseq. Alpha diversity was significantly decreased by HFD diet according to the Chaol and Shannon index $(P<0.05$, Figure $6 \mathrm{~A}, \mathrm{~B})$, which was further increased by mediumand high-dose RSV treatment for Chao1 index (Figure 6A).
According to PCoA, all HFD-fed mice showed a dramatic shift in gut microbiota composition. The RSV treatment group reduced the severity of disturbance in the flora composition in a dose-dependent manner with the medium dose showing the greatest impact. However, all RSV doses did not reverse the changes in intestinal flora caused by HFD (Figure 6C). For the bacterial family, HFD feeding induced significant increase in Desulfovibrionaceae. After treatment with medium and high doses of RSV, the relative levels were not significantly different from normal mice. Prevotellaceae $(P<0.05)$ and Verrucomicrobiaceae $(P<0.05)$ were significantly decreased by HFD feeding. Medium dose of RSV treatment significantly increased Deferribacteraceae, compared with both NC and HFD mice $(P<0.05$, Figure 6D).

\section{Heat map shows the correlation between gut microbiota and body weight}

Although the heat map (Figure 7) shows the correlation of several indicators, we focused primarily on body weight, growth rate, and colonic bacterial content during the experimental analysis. The bacteria included were as follows:

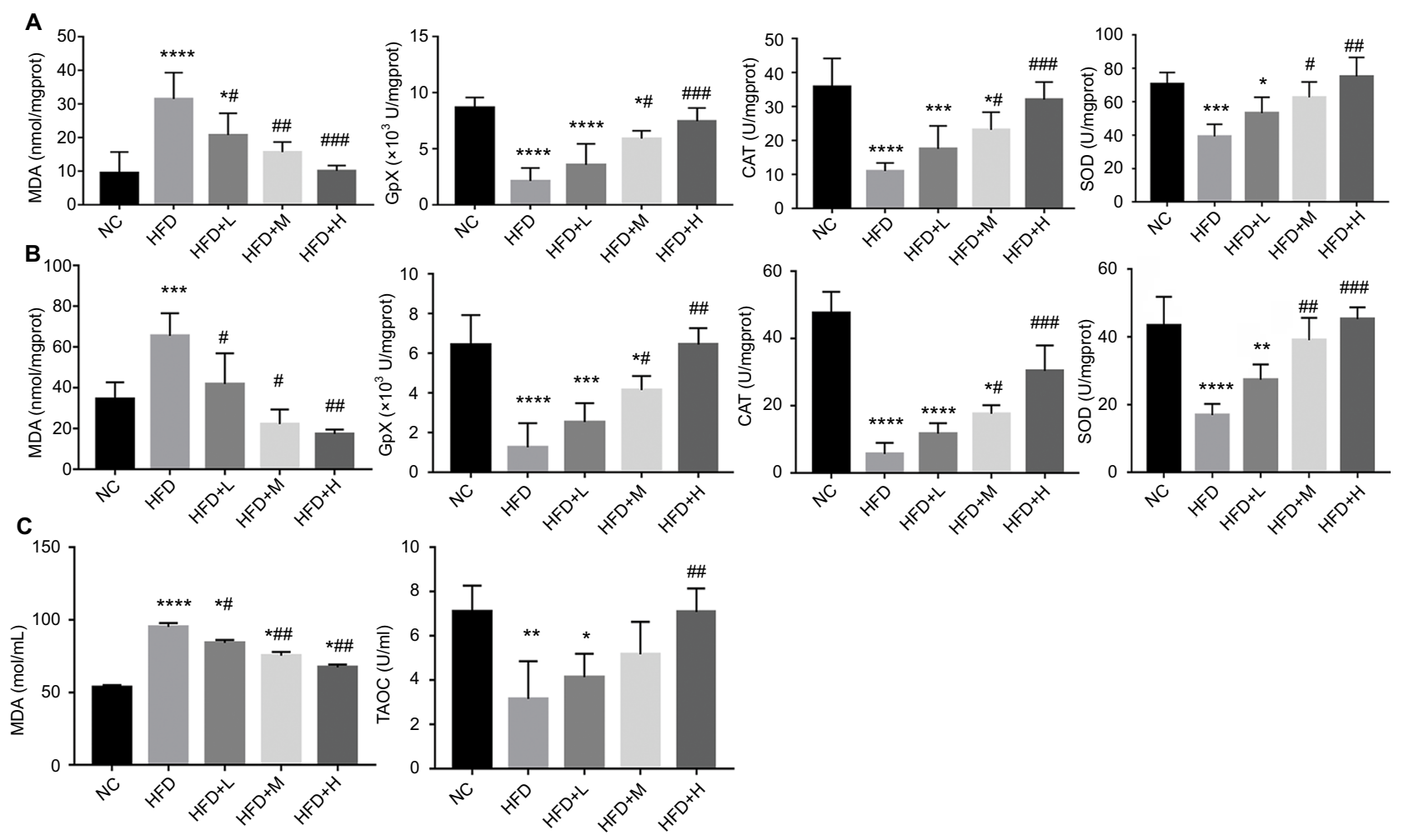

Figure 5 RSV supplement improved the antioxidant and oxidation in HFD-fed mice.

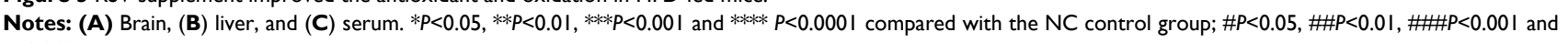
$P<0.000$ I compared with the HFD control group.

Abbreviations: CAT, catalase; GPX, glutathione peroxidase; HFD, high-fat diet; HFD+H, mice fed with HFD supplemented with high RSV; HFD+L, mice fed with HFD supplemented with low RSV; HFD+M, mice fed with HFD supplemented with medium RSV; MDA, malondialdehyde; NC, normal chow diet-fed mice; RSV, resveratrol; SOD, superoxide dismutase; TAOC, total antioxidant capacity. 
A

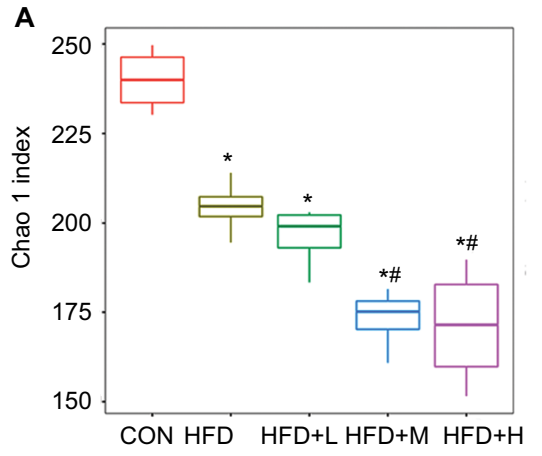

D

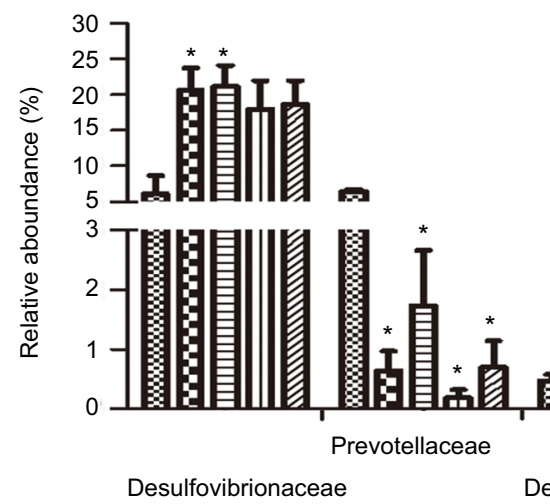

\section{B}

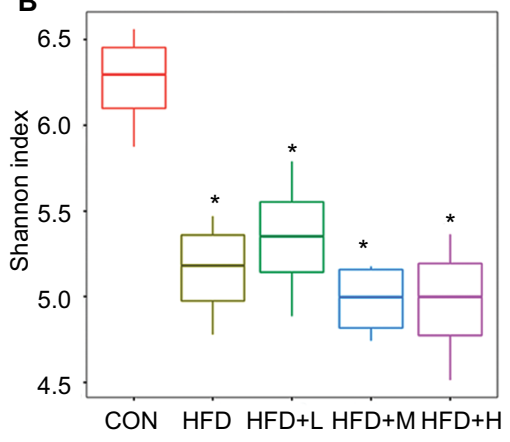$$
\text { . }
$$

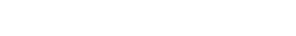

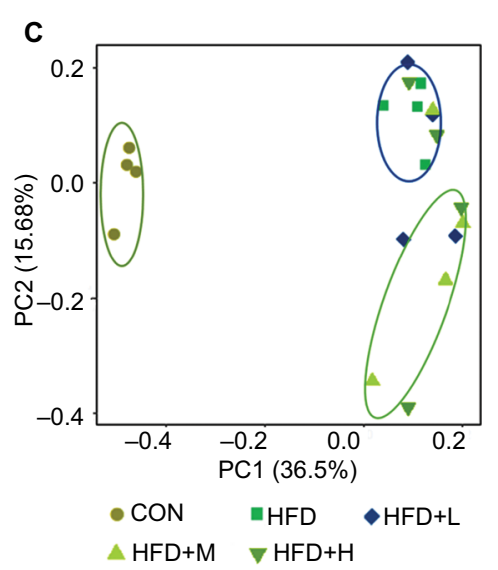

Figure 6 Resveratrol alters the gut microbiota composition in HFD-fed mice. $* P<0.05$ and $* * P<0.01$ compared with the NC control group; \#P<0.05 compared with the HFD control group.

Notes: (A) Chaol, (B) Shannon, (C) principal component analysis (PCA), and (D) relative abundances of the gut microbiota in the feces of mice.

Abbreviations: CON, standard diet; HFD, high-fat diet; HFD+H, mice fed with HFD supplemented with high RSV; HFD+L, mice fed with HFD supplemented with low RSV; HFD+M, mice fed with HFD supplemented with medium RSV; NC, normal chow diet-fed mice; RSV, resveratrol.

Coriobacteriaceae and Desulfovibrionaceae. We observed that increased abundance of Coriobacteriaceae resulted in significant increase in body weight of the HFD mice while Desulfovibrionaceae displayed increased bacterial levels and body adipose mass. This resulted in elevated proliferation of adipose tissue facilitating the development of obesity.

\section{Discussion}

RSV is a polyphenolic compound with anti-obesity effects. However, the exact mechanisms of action remain unclear. ${ }^{28-30}$ Because of very high accumulation in colon tissue but low systemic bioavailability, we hypothesized that through the Illumina Miseq method, RSV might have anti-obesity effects by targeting the gut microbiota, and, in turn, refining metabolism and fat storage. . $^{31,32}$

In our study, we observed that the body weight of mice significantly increased after several weeks of feeding on HFD. However, treatment with RSV resulted in lower body weight gain, growth rate, and liver ratio compared with HFD mice. Mice treated with RSV at doses of 75 and $100 \mathrm{mg} / \mathrm{kg}$ body weight per day showed reduced final body weight and growth rate without significant changes in food intake. ${ }^{33,34}$ These results reveal that RSV has a potential anti-obesity effect. ${ }^{35}$ We observed that epididymal, perididymal, and mesenteric fat pad sizes were smaller in HFD-fed mice supplemented with RSV compared with HFD mice. RSV treatment at $75-100 \mathrm{mg} / \mathrm{kg}$ dose was more effective than the lower dose at suppressing gut fat accumulation. ${ }^{28,36}$

Our results suggested that RSV may be an effective antiobesity agent, decreasing body weight and fat accumulation in response to HFD, and that $75 \%-100 \%$ RSV may be a satisfactory dose for suppressing body weight gain and adiposity. RSV seems to evade the dyslipidemia development in C57BL/6J-treated mice since they prohibited the development of higher LDL-C and lower HDL-C. Surprisingly, high-dose oral RSV supplementation displayed significantly reduced LDL-C levels in HFD mice compared with that in the NC group. These broad range of effects are in agreement with other reports demonstrating the effectiveness of RSV supplementation in body fat reduction. ${ }^{37,38}$ Previous studies have shown that RSV prevented disruption of intestinal flora when administered with HFD. ${ }^{18,21,23}$ In our study, we administered RSV after obesity development in mice, which resulted in failure to restore the gut microbiota altered by 


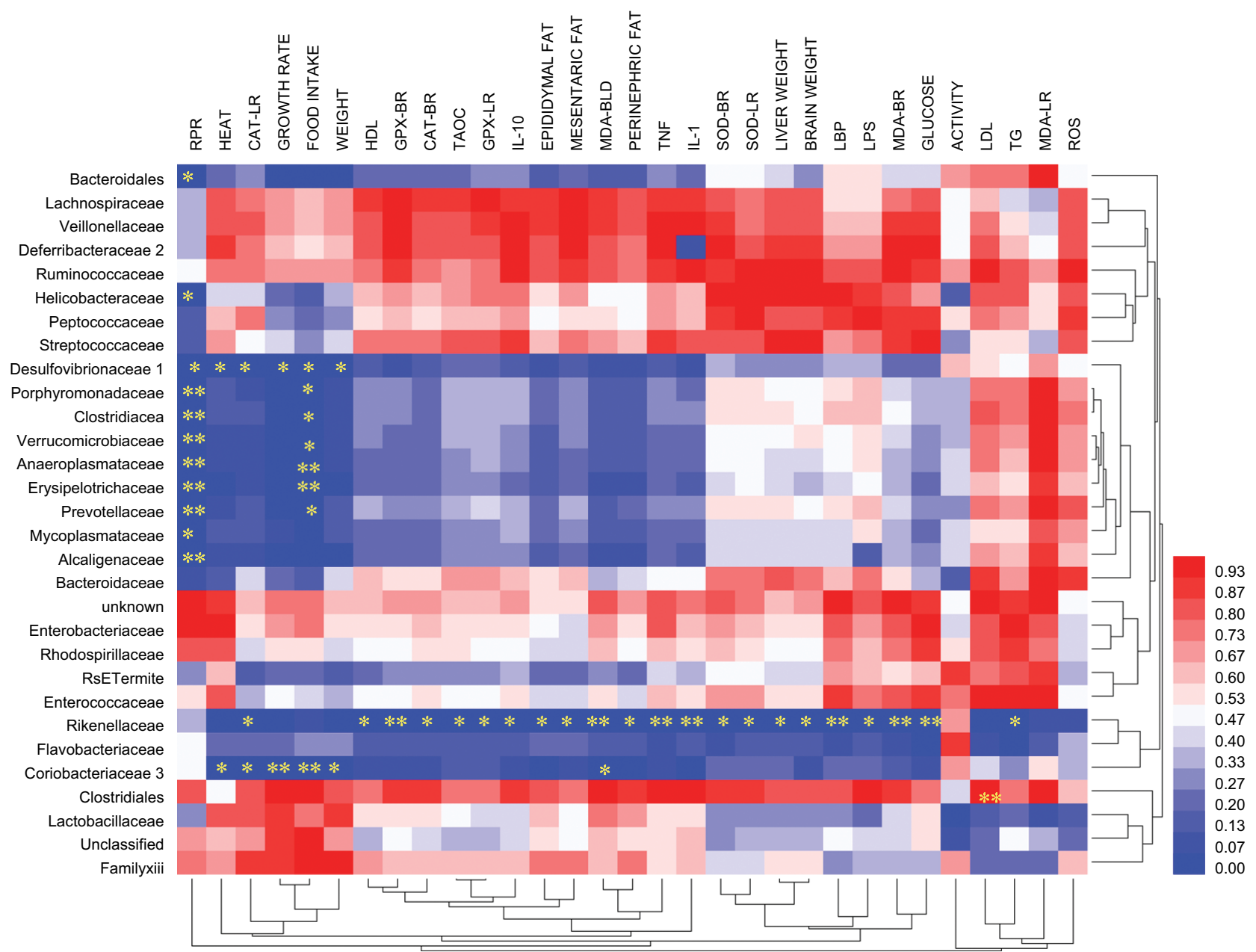

Figure 7 Hierarchical clustering heat map of specific gut bacteria and other biomarkers.

Abbreviations: CAT-LR, catalase in liver; HDL, high-density lipoprotein; GPX-BR, glutathione peroxidase in brain; CAT-BR, catalase in brain; GPX-LR, glutathione peroxidase in liver; IL, interleukin; LBP, lipopolysaccharide-binding protein; LDL, low-density lipoprotein; LPS, lipopolysaccharide; MDA-BLD, malondialdehyde in blood; MDA-BR, malondialdehyde in brain; MDA-LR, malondialdehyde in liver; RPR, rate of perceived recovery; SOD-BR, superoxide dismutase in brain; SOD-LR, superoxide dismutase in liver; TAOC, total antioxidative capacity; TG, triglyceride; TNF, tumor necrosis factor.

HFD. However, the gut barrier was restored by RSV through direct antioxidant effect on the gut.

As plasma TG and blood glucose concentrations were higher in the HF group $(P<0.05)$, RSV significantly decreased the serum TG and blood glucose in HFD after 16 weeks of feeding. ${ }^{36,39}$ Highly significant and persistent effects by RSV in C57BL/6J was also revealed during the experimented period. We also evaluated the anti-oxidative activity of RSV where we observed increased ROS production and/or failure of antioxidant system leading to oxidative stress in the HFD group. HFD mice revealed decrease in activity of SOD, CAT, GSH-Px, and TAOC and increased MDA in blood, liver, and brain while RSV displayed its antioxidant activities by increasing SOD, CAT, GSH-Px, and TAOC and decreasing MDA levels. ${ }^{40,41}$ We found that obesity is companied with inflammation showing increased number of adipose tissue macrophage. ${ }^{42}$ This was confirmed in our study by increased serum TNF-a and Il-1 in HFD mice. RSV showed anti-inflammatory effect by preventing an increase of these cytokines in serum. HFD mice exhibited increase in TNF- $\alpha$, IL-6, and IL-10 levels, while mice receiving different doses of RSV treatment showed some level of differences.

RSV-treated mice displayed significant reduction in the pro-inflammatory profile found in C57BL/6 mice with the highest dose of treatment compared with the HFD group. ${ }^{43,44}$ We assessed the involvement of gut microbiota on the development of metabolic endotoxemia and inflammation. HFD mice displayed higher inflammatory effect in plasma LPS and LBP concentration. Again, RSV displayed its anti-inflammatory effect by reducing plasma LPS and LBP concentration in gut microbes. ${ }^{45,46}$ Increased Firmicutes and decreased Bacteroidetes in feces have strongly been linked to obese mice and diet intake. ${ }^{47}$ 
Bacteroidetes and Firmicutes are key bacterial phyla that have been shown to regulate energy metabolism homeostasis in obesity. ${ }^{48}$ In this study, it was observed that mice fed with HFD showed decreased Bacteroidetes-to-Firmicutes ratios compared with that of NC mice. RSV improved this reaction showcasing its anti-obesity effect on gut microbes. ${ }^{49}$ Our reports emphasized the antimicrobial effects of RSV, which showed a decrease and increase in some of the relative abundance of the following two bacteria species Coriobacteriaceae and Desulfovibrionaceae. These bacterial species have been reported to be closely connected to obesity. ${ }^{18,50}$ Our study also displayed the bacterial profile of the three mentioned relative abundance using Shannon and Chaol analysis. Our results showed decrease of Coriobacteriaceae (actinobacteria-bifidobacteria) in HFD mice compare with NC. Surprisingly, RSV inhibited the growth of Enterococcus faecalis, increasing Coriobacteriaceae bacteria as well as improving Lactobacillus and Bifidobacterium in gut microbiota dysbiosis induced by HFD. ${ }^{50,51}$

Desulfovibrionaceae (Proteobacteria) is associated with inflammation and can cause severe imbalances in the microbiome. Inability to regulate levels of Desulfovibrionaceae within obese individuals has been shown to trigger cecal inflammation, which can lead to local and systemic inflammation subsequently leading to metabolic dysfunction. ${ }^{52,53}$ Our study shows these imbalances in the microbiome, especially in HFD mice compared with others. However, mice that received RSV supplementation had lower Desulfovibrionaceae compared with that in HFD mice. The anti-inflammatory effect of RSV relieved both Lactobacillus and Bifidobacterium levels in gut microbiota dysbiosis induced by HFD. We also used several analyses to examine the relationship between relative abundances of the five groups to know the bacteria level and the impact of RES treatment. Our data show a significant relationship difference with Shannon and Chaol diversity indices.

\section{Conclusion}

Our results suggest that RSV exerts its protective effect on HFD-induced obesity development by integrative responses involving its antioxidant and anti-inflammatory properties. RSV treatment after development of obesity significantly prevented the occurrence of endotoxemia without the restoration of the intestinal microbiota composition. This suggests that it may act directly on the mucosal system to prevent endotoxin migration.

\section{Acknowledgment}

The authors thankfully acknowledge financial support from Wuxi Municipal Science and Education Strengthening Health
Engineering Medical Key Discipline Construction Program (ZDXK003), Wuxi Municipal Science and Education Strengthening Health Engineering Medical Young Talent Project (QNRC039), and Wuxi Municipal Commission of Health and Family Planning Medical Research Project (Q201613).

\section{Disclosure}

The authors report no conflicts of interest in this work.

\section{References}

1. Rokholm B, Baker JL, Sørensen TI. The levelling off of the obesity epidemic since the year 1999--a review of evidence and perspectives. Obes Rev. 2010;11(12):835-846.

2. Jones SE. Chapter 1 - The global problem of obesity. In: Weaver JU, editor. Practical Guide to Obesity Medicine: Elsevier; 2018:1-7.

3. Conway B, Rene A. Obesity as a disease: no lightweight matter. Obes Rev. 2004;5(3):145-151.

4. Surwit RS, Kuhn CM, Cochrane C, McCubbin JA, Feinglos MN. Dietinduced type II diabetes in C57BL/6J mice. Diabetes. 1988;37(9): 1163-1167.

5. Cani PD, Neyrinck AM, Fava F, et al. Selective increases of bifidobacteria in gut microflora improve high-fat-diet-induced diabetes in mice through a mechanism associated with endotoxaemia. Diabetologia. 2007;50(11):2374-2383.

6. Matsuzawa-Nagata N, Takamura $\mathrm{T}$, Ando $\mathrm{H}$, et al. Increased oxidative stress precedes the onset of high-fat diet-induced insulin resistance and obesity. Metabolism. 2008;57(8):1071-1077.

7. Brownlee IA, Knight J, Dettmar PW, Pearson JP. Action of reactive oxygen species on colonic mucus secretions. Free Radic Biol Med. 2007;43(5):800-808.

8. Deitch EA, Xu D, Naruhn MB, Deitch DC, Lu Q, Marino AA. Elemental diet and IV-TPN-induced bacterial translocation is associated with loss of intestinal mucosal barrier function against bacteria. Ann Surg. 1995;221(3):299-307.

9. Burns J, Yokota T, Ashihara H, Lean ME, Crozier A. Plant foods and herbal sources of resveratrol. J Agric Food Chem. 2002;50(11): 3337-3340.

10. Howitz KT, Bitterman KJ, Cohen HY, et al. Small molecule activators of sirtuins extend Saccharomyces cerevisiae lifespan. Nature. 2003;425(6954):191-196.

11. Hüsken A, Baumert A, Milkowski C, Becker HC, Strack D, Möllers C. Resveratrol glucoside (Piceid) synthesis in seeds of transgenic oilseed rape (Brassica napus L). Theor Appl Genet. 2005;111(8):1553-1562.

12. Leonard SS, Xia C, Jiang BH, et al. Resveratrol scavenges reactive oxygen species and effects radical-induced cellular responses. Biochem Biophys Res Commun. 2003;309(4):1017-1026.

13. Su HC, Hung LM, Chen JK. Resveratrol, a red wine antioxidant, possesses an insulin-like effect in streptozotocin-induced diabetic rats. $A m$ $J$ Physiol Endocrinol Metab. 2006;290(6):E1339-E1346.

14. Losa GA. Resveratrol modulates apoptosis and oxidation in human blood mononuclear cells. Eur J Clin Invest. 2003;33(9):818-823.

15. Liu GS, Zhang ZS, Yang B, He W. Resveratrol attenuates oxidative damage and ameliorates cognitive impairment in the brain of senescenceaccelerated mice. Life Sci. 2012;91(17-18):872-877.

16. Rascón B, Hubbard BP, Sinclair DA, Amdam GV. The lifespan extension effects of resveratrol are conserved in the honey bee and may be driven by a mechanism related to caloric restriction. Aging. 2012;4(7):499-508.

17. Delmas D, Lin HY. Role of membrane dynamics processes and exogenous molecules in cellular resveratrol uptake: consequences in bioavailability and activities. Mol Nutr Food Res. 2011;55(8):1142-1153.

18. Etxeberria U, Arias N, Boqué N, et al. Reshaping faecal gut microbiota composition by the intake of trans-resveratrol and quercetin in high-fat sucrose diet-fed rats. J Nutr Biochem. 2015;26(6):651-660. 
19. Chen ML, Yi L, Zhang Y, et al. Resveratrol attenuates trimethylamine- $N$ oxide (TMAO)-induced atherosclerosis by regulating TMAO synthesis and bile acid metabolism via remodeling of the gut microbiota. MBio. 2016;7(2):e02210-02215.

20. Sung MM, Byrne NJ, Robertson IM, et al. Resveratrol improves exercise performance and skeletal muscle oxidative capacity in heart failure. $\mathrm{Am}$ J Physiol Heart Circ Physiol. 2017;312(4):H842-H853.

21. Sung MM, Kim TT, Denou E, et al. Improved glucose homeostasis in obese mice treated with resveratrol is associated with alterations in the gut microbiome. Diabetes. 2017;66(2):418-425.

22. Most J, Penders J, Lucchesi M, Goossens GH, Blaak EE. Gut microbiota composition in relation to the metabolic response to 12 -week combined polyphenol supplementation in overweight men and women. Eur J Clin Nutr. 2017;71(9):1040-1045.

23. Qiao Y, Sun J, Xia S, Tang X, Shi Y, Le G. Effects of resveratrol on gut microbiota and fat storage in a mouse model with high-fat-induced obesity. Food Funct. 2014;5(6):1241-1249.

24. Wu H, Zhang J, Mi Z, Xie S, Chen C, Zhang X. Biofilm bacterial communities in urban drinking water distribution systems transporting waters with different purification strategies. Appl Microbiol Biotechnol. 2015;99(4):1947-1955.

25. Magoč T, Salzberg SL. FLASH: fast length adjustment of short reads to improve genome assemblies. Bioinformatics. 2011;27(21):2957-2963.

26. Caporaso JG, Kuczynski J, Stombaugh J, et al. QIIME allows analysis of high-throughput community sequencing data. Nat Methods. 2010;7(5):335-336.

27. Wang Q, Garrity GM, Tiedje JM, Cole JR. Naive Bayesian classifier for rapid assignment of rRNA sequences into the new bacterial taxonomy. Appl Environ Microbiol. 2007;73(16):5261-5267.

28. Kim S, Jin Y, Choi Y, Park T. Resveratrol exerts anti-obesity effects via mechanisms involving down-regulation of adipogenic and inflammatory processes in mice. Biochem Pharmacol. 2011;81(11):1343-1351.

29. de Ligt M, Timmers S, Schrauwen P. Resveratrol and obesity: can resveratrol relieve metabolic disturbances? Biochim Biophys Acta. 2015;1852(6):1137-1144.

30. Gulvady AA, Ciolino HP, Cabrera RM, Jolly CA. Resveratrol inhibits the deleterious effects of diet-induced obesity on thymic function. $J$ Nutr Biochem. 2013;24(9):1625-1633.

31. Willenberg I, Michael M, Wonik J, Bartel LC, Empl MT, Schebb NH. Investigation of the absorption of resveratrol oligomers in the Caco-2 cellular model of intestinal absorption. Food Chem. 2015;167:245-250.

32. Chiou Y-S, Wu J-C, Huang Q, et al. Metabolic and colonic microbiota transformation may enhance the bioactivities of dietary polyphenols. J Funct Foods. 2014;7:3-25.

33. Alexandre EC, Calmasini FB, de Oliveira MG, et al. Chronic treatment with resveratrol improves overactive bladder in obese mice via antioxidant activity. Eur J Pharmacol. 2016;788:29-36.

34. Gómez-Zorita S, Fernández-Quintela A, Macarulla MT, et al. Resveratrol attenuates steatosis in obese Zucker rats by decreasing fatty acid availability and reducing oxidative stress. Br J Nutr. 2012;107(2): 202-210.

35. Mohammed S, Harikumar KB. Chapter 3 - Role of resveratrol in chemosensitization of cancer. In: Bharti AC, Aggarwal BB, editors. Role of Nutraceuticals in Cancer Chemosensitization. Vol 2: Academic Press;2018:61-76.
36. Lagouge M, Argmann C, Gerhart-Hines Z, et al. Resveratrol improves mitochondrial function and protects against metabolic disease by activating SIRT1 and PGC-1alpha. Cell. 2006;127(6):1109-1122.

37. Louis XL, Thandapilly SJ, Mohankumar SK, et al. Treatment with lowdose resveratrol reverses cardiac impairment in obese prone but not in obese resistant rats. J Nutr Biochem. 2012;23(9):1163-1169.

38. Chen WJ, Du JK, Hu X, et al. Protective effects of resveratrol on mitochondrial function in the hippocampus improves inflammation-induced depressive-like behavior. Physiol Behav. 2017;182:54-61.

39. Palsamy P, Sivakumar S, Subramanian S. Resveratrol attenuates hyperglycemia-mediated oxidative stress, proinflammatory cytokines and protects hepatocytes ultrastructure in streptozotocin-nicotinamide-induced experimental diabetic rats. Chem Biol Interact. 2010;186(2):200-210.

40. Palsamy P, Subramanian S. Resveratrol protects diabetic kidney by attenuating hyperglycemia-mediated oxidative stress and renal inflammatory cytokines via Nrf2-Keap1 signaling. Biochim Biophys Acta. 2011;1812(7):719-731.

41. Soufi FG, Mohammad-Nejad D, Ahmadieh H. Resveratrol improves diabetic retinopathy possibly through oxidative stress - nuclear factor $\kappa \mathrm{B}$ - apoptosis pathway. Pharmacol Rep. 2012;64(6): 1505-1514.

42. Catrysse L, van Loo G. Adipose tissue macrophages and their polarization in health and obesity. Cell Immunol. 2018;330:114-119.

43. Tung BT, Rodríguez-Bies E, Talero E, et al. Anti-inflammatory effect of resveratrol in old mice liver. Exp Gerontol. 2015;64:1-7.

44. Sharma M, Mohapatra J, Wagh A, et al. Involvement of TACE in colon inflammation: a novel mechanism of regulation via SIRT-1 activation. Cytokine. 2014;66(1):30-39.

45. Andrade JMO, Paraíso AF, de Oliveira MVM, et al. Resveratrol attenuates hepatic steatosis in high-fat fed mice by decreasing lipogenesis and inflammation. Nutrition. 2014;30(7-8):915-919.

46. Zhu MJ. Chapter 24 - Dietary polyphenols, gut microbiota, and intestinal epithelial health. In: Bagchi D, Nair S, editors. Nutritional and Therapeutic Interventions for Diabetes and Metabolic Syndrome (Second Edition): Academic Press; 2012:295-314.

47. Turnbaugh PJ. Microbes and diet-induced obesity: fast, cheap, and out of control. Cell Host Microbe. 2017;21(3):278-281.

48. Hyer S. Chapter 7 - the role of human gut microbiota in obesity. In: Weaver JU, editor. Practical Guide to Obesity Medicine: Elsevier; 2018:71-76.

49. Kemperman RA, Gross G, Mondot S, et al. Impact of polyphenols from black tea and red wine/grape juice on a gut model microbiome. Food Res Int. 2013;53(2):659-669.

50. Cardona F, Andrés-Lacueva C, Tulipani S, Tinahones FJ, Queipo-Ortuño MI. Benefits of polyphenols on gut microbiota and implications in human health. J Nutr Biochem. 2013;24(8):1415-1422.

51. Jiao X, Wang Y, Lin Y. Blueberry polyphenols extract as a potential prebiotic with anti-obesity effects on $\mathrm{C} 57 \mathrm{BL} / 6 \mathrm{~J}$ mice by modulating the gut microbiota. J Nutr Biochem. 2018.

52. Boutagy NE, McMillan RP, Frisard MI, Hulver MW. Metabolic endotoxemia with obesity: Is it real and is it relevant? Biochimie. 2016;124: 11-20.

53. Carvalho FA, Koren O, Goodrich JK, et al. Transient inability to manage proteobacteria promotes chronic gut inflammation in TLR5-deficient mice. Cell Host Microbe. 2012;12(2):139-152.

Diabetes, Metabolic Syndrome and Obesity: Targets and Therapy

\section{Publish your work in this journal}

Diabetes, Metabolic Syndrome and Obesity: Targets and Therapy is an international, peer-reviewed open-access journal committed to the rapid publication of the latest laboratory and clinical findings in the fields of diabetes, metabolic syndrome and obesity research. Original research, review, case reports, hypothesis formation, expert opinion and commentaries are all considered for publication. The manuscript management system is completely online and includes a very quick and fair peer-review system, which is all easy to use. Visit http://www.dovepress.com/testimonials.php to read real quotes from published authors. 